\title{
ARTIGOS
}

Submetido 14.02.2017. Aprovado 14.07.2017

Avaliado pelo sistema double blind review. Editor Científico Convidado: Cláudio Lucinda

Versão original

DOI: http://dx.doi.org/10.1590/So034-759020180406

\section{CORRUPÇÃO GOVERNAMENTAL NO MERCADO DE CAPITAIS: UM ESTUDO ACERCA DA OPERAÇÃO LAVA JATO}

\author{
Government corruption on Brazilian capital markets: A study on Lava Jato (Car \\ Wash) investigation \\ Corrupción gubernamental en el mercado de capitales: Un estudio acerca de la \\ operación Lava Jato
}

\section{RESUMO}

O Brasil apresentou, nos últimos anos, um cenário de crise econômica e política, resultado do funcionamento de uma elaborada rede de corrupção no governo. A literatura financeira aponta duas linhas de pensamento relacionadas ao tema corrupção, uma que defende a existência de efeitos benéficos e outra que aponta os prejuízos no sistema público. A fim de se entender a relação entre a queda no nível de investimento e a corrupção, foi realizado um estudo de eventos, com base no modelo GARCHX-in- Mean, que permitisse identificar a existência e/ou o potencial sentido do impacto da corrupção para alguns ativos selecionados. Os resultados apontam que, no Brasil, houve uma grande desvalorização nas maiores empresas estatais negociadas na bolsa de valores nacional e também em suas American Depositary Receipt (ADR), consequentemente, gerando um cenário de fuga de recursos financeiros e indicando uma queda no crescimento econômico, apoiando a teoria de prejuízo em longo prazo da corrupção.

PALAVRAS-CHAVE | Estudo de eventos, retorno, volatilidade, GARCHX-in-mean, corrupção.

\section{ABSTRACT}

In recent years, Brazil has faced economic and political crises resulting from the actions of an elaborate syndicate of corruption in the government. The financial literature points to two lines of thought related to corruption. One line defends the existence of benefits and the other points to losses in the public network. An event study based on the GARCHX-in-Mean model was conducted to understand the relationship between corruption and the decrease in the level of investment, and identify the potential impact of corruption on selected assets. The results indicate that there was a significant devaluation of the largest state-owned companies traded on the Brazilian Stock Exchange and in American Depositary Receipts, leading to money laundering and a decrease in economic growth. This supports the theory of long-term crisis due to corruption.

KEYWORDS / Event study, return, volatility, GARCHX-in-mean, corruption.

ANA JULIA AKAISHI PADULA ${ }^{1}$

anajulia.padula@gmail.com

ORCID: 0000-0002-2969-1939

\section{PEDRO HENRIQUE MELO} ALBUQUERQUE

\section{pedroa@unb.br}

ORCID: 0000-0002-1415-716X

${ }^{1}$ Universidade de Brasília, Departamento de Administração, Brasília, DF, Brasil

\section{RESUMEN}

Brasil presentó, en los últimos años, un escenario de crisis económica y política, resultado del funcionamiento de una elaborada red de corrupción en el gobierno. La literatura financiera apunta dos líneas de pensamiento relacionadas al tema corrupción: una que defiende la existencia de efectos benéficos y otra que apunta los perjuicios en el sistema público. A los efectos de entender la relación entre la caída del nivel de inversión y la corrupción, se realizó un estudio de eventos, con base en el modelo GARCHX-in-mean, que permitiera identificar la existencia y/o el potencial sentido del impacto de la corrupción para algunos activos seleccionados. Los resultados indican que, en Brasil, hubo una gran desvalorización en las mayores empresas estatales negociadas en la bolsa de valores nacional y también en sus American depositary receipt $(A D R)$ que, consecuentemente, generó un escenario de fuga de recursos financieros e indicó una baja en el crecimiento económico, lo que apoya la teoría de perjuicio a largo plazo de la corrupción.

PALABRAS CLAVE / Estudio de eventos, rendimiento, volatilidad, GARCHX-in-mean, corrupción. 


\section{INTRODUÇÃO}

A economia mundial apresentou uma grande mudança na última década. Com o avanço da tecnologia, o efeito da globalização mostra-se cada vez mais forte e presente no cotidiano. A conectividade entre os países e o fácil acesso às informações permitiram um aumento de transações internacionais em diversos mercados. Claessens, Klingebiel e Schmukler (2006), por exemplo, discutem que a integração e negociação de bens e recursos entre os países impacta os agentes envolvidos em sua estrutura econômica e legal a fim de manter a competitividade nacional e ainda garantir o crescimento comercial.

O Brasil apresentou uma grande mudança em sua estrutura social e econômica, como aponta o World Bank (2015). O País, que apresentava um Produto Interno Bruto (PIB) médio de 4,5\% entre 2006 e 2010, apresentou uma queda nos anos seguintes, devido à crise instaurada nos setores econômico e político, como resultado da corrupção pública, chegando a $-3,05 \%$, segundo o relatório de outubro de 2015 do Banco Central do Brasil (2015). O cenário de incerteza e desconfiança provocado pelo alto gasto governamental e o aumento da inflação e da taxa de juros geraram um cenário de dificuldade para as empresas brasileiras sobreviverem e crescerem, resultado observado na alteração do retorno e aumento do risco dos ativos no mercado financeiro.

Diversos estudos ao redor do mundo, como os de Shleifer e Vishny (1993) e Mauro (1995), buscaram entender a relação entre a corrupção governamental e o crescimento do país. Shleifer e Vishny (1993) definiram esse fenômeno como o uso do poder e da influência pública para ganhos pessoais, sendo mais encontrado em países em desenvolvimento, devido à estruturação comum desses governos, instáveis e extremamente burocráticos. De maneira geral, as pesquisas indicam efeitos positivos e negativos do evento corrupção no desenvolvimento de cada nação, criando duas correntes de análise: (1) Grease the Wheel, que apresenta os resultados benéficos que a corrupção possui na economia; e (2) Sand the Wheel, que critica a corrupção e busca evidenciar os obstáculos e prejuízos causados por ela. Dentro das análises, são considerados diversos pontos, sociais e econômicos, que buscam identificar as causas e fatores que contribuem para a continuidade do evento em questão. Ainda assim, poucos são os estudos voltados especificamente para os efeitos em um país, quanto mais para a análise socioeconômica dessa relação.

Como prova do atual e crescente interesse pela corrupção pública, diversas entidades internacionais abriram espaços para discussão sobre o tema. Em janeiro de 2017, foi realizada em Davos, na Suíça, a reunião do World Economic Forum, uma organização que tem por objetivo discutir diversos pontos sobre o desenvolvimento econômico e social dos países envolvidos, sendo um dos tópicos o combate à corrupção e os esforços de cada nação para combatê-la. A agenda de debate apresentou a forte e constante presença da corrupção na América Latina, e como países como Brasil e Chile apresentaram uma postura proativa e compromissada com investigações e punições aos envolvidos em escândalos de corrupção, tópico que tem chamado a atenção da mídia internacional, especialmente, desde 2014.

Ao considerar a atenção e o destaque que as comunidades internacional e nacional demonstram sobre o tema, investigações como a Operação Lava Jato apresentam um caráter fundamental para entender e quantificar o impacto que a corrupção possui no País. A Lava Jato foi iniciada em 17 de março de 2014, com o objetivo de apurar crimes financeiros e de desvio de recursos públicos, e possui, atualmente, 30 fases de investigação, sendo classificada no World Economic Forum como um dos maiores escândalos de corrupção já denunciados no Brasil. Inicialmente, as investigações buscavam apenas desvendar crimes envolvidos com operações ilícitas em postos de gasolina, porém, com o avanço das procuras, novas provas foram obtidas, e um grande esquema de corrupção, que englobou grandes setores da economia, tais como: petróleo, construção civil, setor financeiro e a própria esfera política do País, foi revelado. Uma vez identificada a extensão da corrupção no sistema socioeconômico do Brasil, o estudo sobre esse evento foi direcionado, em um primeiro momento, para ativos presentes no mercado de capitais e que apresentam grande relevância na composição do principal índice da Bolsa de Valores Mercadorias e Futuros (BM\&FBOVESPA), sendo estes: Petrobras (PETR4 e PETR3), Vale (VALE5 e VALE3) e Banco do Brasil (BBAS3). A escolha das empresas estatais deveu-se pela forte presença dos ativos no mercado de capitais nacional e por representarem os setores de petróleo, mineração e financeiro do País, além de estarem ligadas diretamente a decisões e eventos que envolvem o Estado brasileiro. Em seguida, o mesmo evento foi analisado para os ativos cotados no mercado internacional na bolsa Nasdaq (s.d.), considerando as mesmas empresas estudas para o mercado brasileiro, Petrobras (PBR.A e PBR), Vale (VALE.P e VALE) e Banco do Brasil (BDORY), por meio de suas ADRs.

Assim, este estudo apresenta uma pesquisa voltada para a realidade brasileira e busca medir, até aonde é possível, qual o impacto que a corrupção possui sobre o risco e o retorno dos principais ativos estatais que compõem o índice da BM\&FBOVESPA. Analisou-se, portanto, como evento focal, o efeito da divulgação das atividades públicas da Operação Lava Jato, tais como: buscas e apreensão, condução coercitiva e prisões, sobre o prêmio pelo risco e volatilidade desses ativos, 
especificamente para as estatais: Petrobras, Banco do Brasil e Vale. A compreensão de como esse evento afeta o prêmio pelo risco e sua volatilidade serão um auxílio para que os agentes econômicos possam reavaliar suas carteiras, além de possibilitar um suporte para políticas de compliance nas organizações do Brasil e na América Latina, por meio da apresentação de como a presença da corrupção institucional é prejudicial em longo prazo, independentemente das aparentes vantagens no curto prazo.

\section{REFERENCIAL TEÓRICO}

Esta seção apresenta as duas principais correntes de pensamento discutidas por pesquisadores quando se trata do tema corrupção pública. Primeiro, serão apresentados resultados e teorias da linha de pesquisa que traz pontos positivos da corrupção, intitulada Grease the Wheels, seguida pela segunda linha de pesquisa, que busca apontar os efeitos negativos do evento, identificada como Sand the Wheels.

\section{Grease the Wheels}

Diversas pesquisas foram realizadas dentro desse campo para tentar comprovar a existência de efeitos benéficos da corrupção no desenvolvimento social e econômico do país. A principal base de argumentação dessa corrente é a presença de um sistema governamental burocrático e ineficiente. Leys (1965) argumenta, em seu trabalho, que uma visão negativa sobre a corrupção contamina a opinião dos demais, devido a princípios moralistas impostos pela sociedade, apesar de a corrupção apresentar um caráter neutro e que se manifesta de acordo com as condições do ambiente.

Huntington (2006) realizou uma análise do sistema burocrático e político de diversos países em momentos de sua história. Ao encontrar um histórico de governos altamente centralizados e burocráticos, o autor diagnosticou um processo público lento e exaustivo para o meio social e econômico. Eventos-chave apresentaram um alto desenvolvimento do país, acompanhado de notícias e escândalos de corrupção, o que leva a acreditar nessa última como um evento balizador para o sistema vagaroso. Seguindo esse princípio, Lau, Demir e Bilgin (2013), em seu estudo, encontraram um efeito positivo em relação à velocidade do governo, na qual o pagamento de subornos e propinas é importante e garante que os servidores públicos realizem seu trabalho e continuem a exercer sua função.

Ao analisar o ganho existente para os servidores públicos, a corrupção em si manifesta um caráter de ganho e proteção para os envolvidos. De acordo com as pesquisas realizadas por Leys (1965) e Bayley (1966), a presença desse fenômeno na esfera pública aumenta a procura de cargos que participem do ciclo de recebimentos de subornos e propinas, sendo, assim, selecionados apenas os indivíduos com aptidão e alta qualificação, além de ser uma garantia de que receberão por seus trabalhos, uma vez que o setor privado está coberto de incertezas e riscos. Analisado esse ponto, Dobson e RamloganDobson (2012) realizaram um estudo verificando o impacto da corrupção no crescimento do setor informal e encontraram uma relação negativa entre as variáveis.

Quando considerada a agilidade fornecida pela corrupção, além dos ganhos citados, o setor de projetos públicos também é beneficiado. Leff (1964) e Bayley (1966) argumentam que existem dois ganhos para o governo e a população: (1) licitações públicas para obras serão mais eficientes, uma vez que as empresas envolvidas devem se apresentar mais competentes para realizar o projeto e entregá-lo com a qualidade acordada, uma vez que uma parte da remuneração que a empresa receberia foi utilizada no pagamento de propina ou suborno para garantir o edital; (2) ao se deparar com uma lei que não possa ser contornada, ou ao receber para garantir o interesse de outrem, o governo faria uma avaliação e revisão das normas existentes; desse modo, leis que não se adequem mais à realidade da sociedade serão alteradas e substituídas.

\section{Sand the Wheels}

Enquanto a corrente Grease the Wheels possui sua base na ineficiência governamental, a corrente Sand the Wheels analisa e contra-argumenta os pontos levantados com base nos custos gerados ao país e nos impactos no longo prazo. Pesquisas realizadas recentemente e apresentadas neste artigo ajudam a aumentar a base desse campo e agregam novos dados do funcionamento real e dos efeitos da corrupção em índices econômicos.

Myrdal (1994) critica a relação existente entre o aumento da velocidade na execução dos processos públicos e a corrupção. No primeiro momento, são levantados dois pontos com relação à lentidão do sistema, que pode ser vagaroso ou aparentar ser vagaroso. No primeiro caso, existe o ganho em curto prazo com a presença da corrupção, porém, quando analisado um horizonte maior de tempo, a evolução do sistema rumo à eficiência será bloqueada pelos fornecedores de serviço. 0 mesmo se aplica ao segundo caso: funcionários públicos terão a tendência de manter e preservar as dificuldades das atividades realizadas para garantir 
o recebimento ilícito, apresentando, assim, um obstáculo para o crescimento do país.

Avaliada a questão sistemática, Kurer (1993) analisou os impactos com relação aos membros dos ciclos e esquemas de corrupção. Em sua pesquisa, o autor descreve o efeito do clientelismo, caracterizado como uma maneira pela qual o integrante mais poderoso e influente mantém seu status com base em aliança e favores em troca de proteção. Esse sistema é identificado dentro da máquina pública, na qual existem círculos que impedem a entrada de novos membros para manter a organização e distribuição atual de ganhos. Desse modo, a entrada de funcionários no setor público não necessariamente resultará em um aumento da qualificação da mão de obra ou na qualidade do serviço, mas, sim, na manutenção ou propagação do sistema já corrupto e ineficiente.

Quanto aos ganhos obtidos nos projetos governamentais, diversos autores criticam e contra-argumentam os pontos expostos pela outra corrente. Primeiramente, Rose-Ackerman (1997) aponta que a presença de pagamentos ilícitos para escolha em editais não significa a escolha do mais eficiente, mas sim daquele que está disposto a desembolsar mais para adquirir a licença; o resultado é uma alocação dos recursos públicos com pouco retorno social, alto custo, qualidade duvidosa, além de apresentar um método de entrada de novas empresas, que apenas participam do esquema de pagamento, sem que necessariamente sejam as melhores. Os autores Bardhan (1997) e Lambsdorff (2003) analisam a reformulação legislativa proposta pela corrupção; a base para a contestação está na característica de a corrupção reduzir o nível de confiança das empresas nas leis. Observa-se que a presença desse fenômeno aumenta o risco percebido pelos empresários no país e em seu funcionamento. Desse modo, o governo não possuiria meios de demonstrar ou garantir legitimidade do seu sistema além do cumprimento de leis.

Além dos pontos inerentes ao funcionamento público, Mauro (1995) analisa o impacto dentro do nível de investimento do país e no crescimento em si. O autor considera, em sua pesquisa, duas variáveis para avaliar corrupção: (1) eficiência da burocracia: medida pelo sistema judicial, quantidade de normas e índices de corrupção; (2) estabilidade política: calculada por meio da mudança institucional, mudanças sociais, controle da oposição, estabilidade do trabalho, relação com países vizinhos e índice de terrorismo. Uma forte relação positiva foi encontrada entre eficiência burocrática e estabilidade política, sendo o principal fator de decisão para investimento privado em um país sua estabilidade política e sua confiança no governo. Ciocchini, Durbin e Ng (2003) buscaram identificar o impacto da corrupção no mercado financeiro, como realizado por Mauro
(1995), mas focados apenas no setor privado. Entre os resultados, identificou-se que os investidores esperam um prêmio pelo risco maior em países que apresentam altos índices de corrupção, sendo esse efeito mais evidente em países emergentes.

Estudo realizado por Nguyen e Dijk (2012) no Vietnã buscou entender a corrupção e seu impacto no país. Os autores apontam seis fatores que propiciam o surgimento do fenômeno e que favorecem sua continuidade: (1) tipo de governança existente, referente ao nível de centralização do governo; (2) autonomia regional, ligada ao poder que cada região possui de exercer suas leis; (3) gastos públicos; (4) acesso à propriedade, considerando o gasto necessário para se ter acesso e o tempo gasto; (5) implementação e consistência de leis, qual o lastro que a lei possui e os argumentos envolvidos para sua criação; e (6) políticas de desenvolvimento do setor privado, sendo este divido em custos de entradas e tempo para entrada no mercado.

Entre os resultados encontrados por Nguyen e Dijk (2012), notou-se que os fatores que reduzem o nível de corrupção no país são: a capacidade de implementação e consistência de leis, além do acesso à propriedade. Esses pontos são decorrentes de um baixo nível de burocratização e alta eficiência do governo; a mesma pesquisa ainda identificou que fatores referentes a políticas de desenvolvimento do setor privado apresentam uma forte relação positiva com o desenvolvimento do evento em questão. Segundo os autores, a relação pode ser explicada devido à burocracia que retarda ao máximo o processo de funcionamento para as empresas privadas.

A literatura hoje apresenta poucos estudos ligados diretamente à realidade de um país ou a um caso comprovado de corrupção. O Brasil já apresenta interesse em estudar esse fenômeno, porém são escassos os trabalhos associados a esse tema e que abordem a ótica do mercado financeiro e de capitais por meio de uma metodologia quantitativa, gap esse suprido por este estudo.

\section{METODOLOGIA}

A metodologia base escolhida para analisar o impacto da corrupção nos mercados financeiro e de capitais possui sua origem do campo da econometria financeira, sendo classificada como estudo de eventos. Segundo Campbell, Lo e MacKinlay (1997), essa técnica foi desenvolvida com o objetivo de entender, mensurar e relacionar o efeito de um determinado evento ou fenômeno no mercado. 0 primeiro estudo publicado a respeito é datado de 1933, por Dolley, em sua análise sobre o retorno da emissão de novas ações em empresas já existentes. Binder 
(1998) discute, em seu trabalho, a evolução e sofisticação da metodologia, com a qual diversos pesquisadores contribuíram para a concepção atual que possuímos do modelo.

Campbell et al. (1997) estipularam as principais etapas para a condução da metodologia de estudo de eventos, sendo uma grande referência para pesquisadores no campo. A primeira etapa é considerada a definição do evento de interesse, na qual se deve definir o fenômeno a ser estudado, o período em que ocorre e estipular, assim, sua base de entradas de acordo com a data; não existem restrições de espaço de tempo, ou seja, a metodologia pode ser aplicada em dados anuais, mensais ou diários, contanto que o número de observações seja suficiente para atender aos pressupostos do modelo (como o Teorema Central do Limite nos casos assintóticos). Alguns autores, como Dann (1981), questionam a aplicabilidade da metodologia em observações diárias, devido à distorção do pressuposto de normalidade no componente estocástico do modelo, efeito que pode ser suavizado ao calcularmos o logaritmo natural do retorno, alterando a distribuição definida no modelo para alguma distribuição com caudas mais pesadas, como feito por Rachev e Mittnik (2000), ou utilizando uma metodologia de estimação dos parâmetros robusta a desvios na normalidade dos dados, como realizado por White (1982).

Considerou-se uma unidade temporal diária para a análise do evento e dos retornos dos ativos em questão. Escolheu-se, ainda, como proxy para o evento de corrupção a divulgação oficial dos desdobramentos da Operação Lava Jato, datas que foram marcos importantes para estabelecer os períodos de análise. Nesse sentido, o primeiro passo é definir as janelas de estimação e de evento, metodologicamente apontadas como período sem a interferência do evento, sendo (1) a janela de estimação para os dados brasileiros de 3 de janeiro de 2000 até 16 de março de 2014 (2) e a janela de estimação para os dados norte-americanos de 13 de junho de 2006 até 16 de março de 2014. O segundo passo é definir a janela do evento, ou seja, o período no qual o evento estudado é encontrado, sendo este datado de 17 de março de 2014 até 29 de dezembro de 2016 e aplicado para ambos os cenários, nacional e internacional.

Estabelecidos os intervalos das janelas, procedeu-se à avaliação do impacto dos eventos associados à Operação Lava Jato, quais sejam buscas e apreensões, conduções coercitivas e prisões. A data de cada desdobramento da operação foi obtida diretamente no site da Polícia Federal (2014), e, para entender a difusão da absorção e difusão das notícias, foram criadas janelas temporais de um, dois, cinco, 10 e 15 dias a partir dessa divulgação. Cada janela representa uma variável binária que assume valor igual a 1 durante o período considerado e zero, caso contrário, resultando em uma base com aparecimentos ou não diários do evento.

0 modelo econométrico adotado para o estudo de evento foi o modelo GARCHX-in-mean (GARCHX-M), escolhido com base nos trabalhos dos autores Jong, Kemna e Kloek (1992), o qual incorpora a presença de volatilidade e dependência temporal na equação da média, e de Staikouras (2006), que introduz as variáveis exógenas na equação da volatilidade. Matematicamente, o modelo adotado assumiu a seguinte estrutura:

$$
\left\{\begin{array}{l}
r_{i t}-r_{f t}=\beta\left(r_{m t}-r_{f t}\right)+\delta I_{j t}+\epsilon_{t} \\
\sigma_{t}^{2}=\gamma_{0}+\gamma_{0}+\gamma_{1} r_{i(t-i)}^{2}+\gamma_{2} \sigma_{i(t-i)}^{2}+\omega I_{j t}
\end{array}\right.
$$

tal que $\epsilon_{t} \sim N\left(0, \sigma_{t}^{2}\right)$. Ademais, considerou-se $r_{i t}$ o retorno ajustado da firma $i$ no período $t$ tal que $i=1,2,3$ representando, respectivamente: Petrobras, Banco do Brasil e Vale. $r_{f t}{ }^{\wedge} r_{m t}$ são respectivamente os retornos do ativo livre de risco, taxa referencial do Sistema Especial de Liquidação e de Custódia (Selic), e o retorno ajustado do índice de mercado (Índice Bovespa) no período $t$ para o mercado nacional. Para o mercado internacional, considerou-se como retorno de mercado o retorno ajustado do índice $S \& P 500$ e como ativo livre de risco Treasury Bills do mercado americano.

Note que, no sistema apresentado pelas Equações, em 1, a primeira equação é composta pelo modelo Capital Asset Pricing Model (CAPM), no qual o prêmio pelo risco $\left(r_{m t}-r_{f t}\right)$ é explicado pelo $\beta$ de mercado multiplicado pelo prêmio pelo risco no mercado $\left(r_{m t}-r_{f t}\right)$. Ademais, o parâmetro $\delta$ mensura o efeito da divulgação do evento sobre o prêmio pelo risco do ativo e mede, portanto, a perda esperada percentual (ou ganho esperado percentual) quando novos desdobramentos do evento de interesse ocorrem.

Na segunda equação, temos o modelo $\operatorname{GARCHX}(1,1)$ para modelar a volatilidade do prêmio pelo risco $\left(r_{m t}-r_{f t}\right)$ somado ao efeito do evento sobre a volatilidade, dado pelo parâmetro $\omega$. A estrutura $\operatorname{GARCH}(1,1)$ foi adotada considerando o comum fato estilizado do bom ajuste desse modelo em séries temporais financeiras, conforme apontado por Hansen e Lunde (2005). Os parâmetros apresentados no sistema de equações 1 foram estimados por meio de Quasi Maximum Likelihood Estimation (QMLE), método robusto a desvios no pressuposto de normalidade, como apresentado por White (1982). 
Em outras palavras, o estimador QMLE é utilizado para modelos cujas distribuições possam apresentar desvios acercas do pressuposto base. De acordo com o modelo apresentado no sistema de equações 1 , esse pressuposto é o pressuposto de normalidade, uma vez que $\in_{t} \sim N\left(0, \sigma_{t}{ }^{2}\right)$. Assim, o estimador QMLE consistentemente estima o vetor de parâmetros de interesse, minimizando a divergência de Kullback-Leibler entre a distribuição postulada e a especificada. Esse estimador, portanto, é assintoticamente normal e consistente, possibilitando boas estimativas, uma vez que a amostra seja suficientemente grande (Cox \& Reid, 2004).

As estimativas para os demais parâmetros do modelo foram omitidas, por não serem os principais parâmetros a serem analisados; entretanto, para todos os modelos estimados, o teste ponderado de Ljung-Box, desenvolvido por Ljung e Box (1978), para o quadrado dos resíduos apresentou $p$-valor superior a $5 \%$, indicando, assim, que a hipótese nula de ausência de correlação serial dos resíduos não deve ser rejeitada, portanto a estrutura de dependência temporal foi complementarmente modelada pelo sistema de equações 1 em todos os casos.

A variável dummy $I_{j t}$ assumiu valor igual a 1 nas datas em que a operação foi realizada, seguida das janelas de $j=\{1,2,5,10$,
$15\}$ posteriores ao registro. Assim como relatado anteriormente, $\delta$ e $\omega$ representam o efeito da divulgação dessa informação nas janelas consideradas para o prêmio pelo risco e volatilidade desse, respectivamente. Espera-se, ainda, que, à medida que essa janela aumente, esse efeito diminua, representando a taxa de "perda de memória" dos investidores, uma vez que, quanto mais distante da data do desdobramento, menor seria esse impacto no prêmio pelo risco e volatilidade.

\section{RESULTADOS}

\section{Mercado nacional}

O impacto do evento, assim como sua dispersão ao longo do tempo, foi mensurado individualmente para cada ativo, considerando os intervalos do aparecimento das notícias oficiais ligadas à Operação Lava Jato e divulgadas no site da Polícia Federal (2014). Os resultados são descritos nas tabelas e apresentam o comportamento do prêmio pelo risco em relação ao evento quando mensuramos a volatilidade e o valor esperado para cada espaço de tempo das notícias de corrupção:

\section{Tabela 1. Resultados sem intervalo pós-divulgação das notícias}

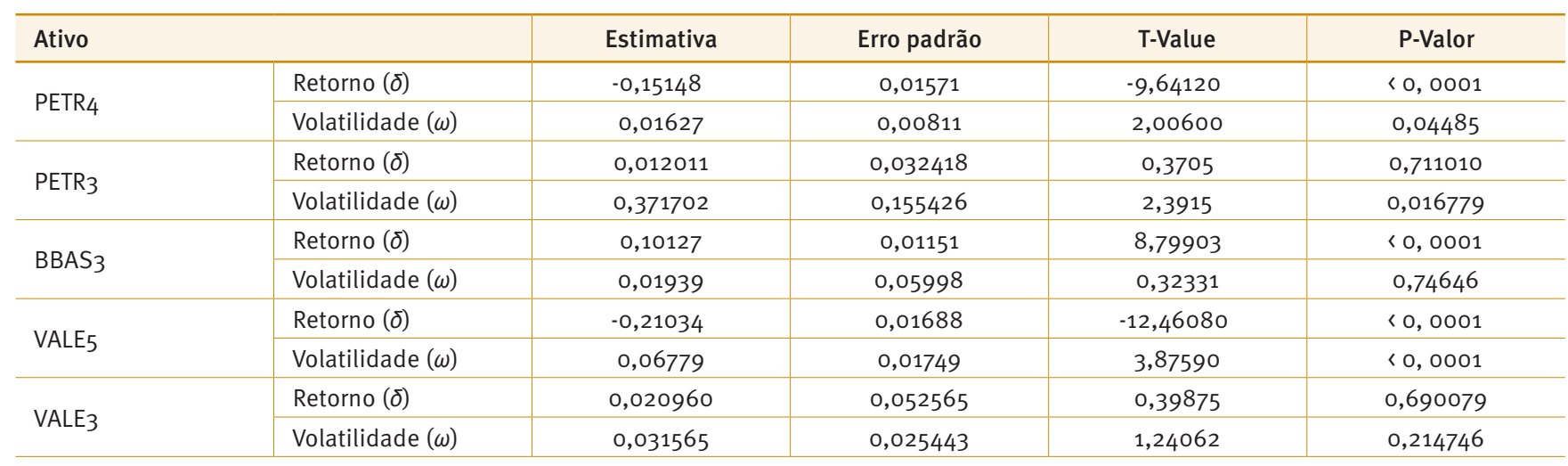

Tabela 2. Resultados com intervalo de dois dias pós-divulgação das notícias

\begin{tabular}{l|l|c|c|c|c}
\hline \multicolumn{1}{l}{ Ativo } & Estimativa & Erro padrão & T-Value & P-Valor \\
\hline \multirow{2}{*}{ PETR4 } & Retorno $(\delta)$ & $-0,16810$ & 0,01128 & $-14,90430$ & $<, 0001$ \\
\cline { 2 - 6 } & Volatilidade $(\omega)$ & 0,02976 & 0,00843 & 3,53060 & 0,0001 \\
\hline \multirow{2}{*}{ PETR3 } & Retorno $(\delta)$ & 0,003109 & 0,040926 & 0,075977 & 0,939438 \\
\cline { 2 - 6 } & Volatilidade $(\omega)$ & 0,417077 & 0,166138 & 2,510422 & $<, 012059$ \\
\hline \multirow{2}{*}{ BBAS3 } & Retorno $(\delta)$ & 0,10971 & 0,02332 & 0,70443 & 0,43049 \\
\cline { 2 - 6 } & Volatilidade $(\omega)$ & 0,19851 & 0,25180 & $-1,10178$ & 0,27056 \\
\hline \multirow{2}{*}{ VALE5 } & Retorno $(\delta)$ & $-0,22058$ & 0,20020 & 0,45675 & 0,64786 \\
\cline { 2 - 6 } & Volatilidade $(\omega)$ & 0,09728 & 0,21299 & $-16,2766$ & 1,7896 \\
\hline \multirow{2}{*}{ VALE3 } & Retorno $(\delta)$ & $-0,178937$ & 0,010994 & 0,0001 \\
\cline { 2 - 6 } & Volatilidade $(\omega)$ & 0,430601 & 0,240608 & 0,073512 & \\
\hline
\end{tabular}


Tabela 3. Resultados com intervalo de cinco dias pós-divulgação das notícias

\begin{tabular}{|c|c|c|c|c|c|}
\hline Ativo & & Estimativa & Erro padrão & T-Value & P-Valor \\
\hline \multirow{2}{*}{ PETR4 } & Retorno $(\delta)$ & $-0,16997$ & 0,01195 & $-14,22102$ & $<0,0001$ \\
\hline & Volatilidade $(\omega)$ & 0,02659 & 0,02465 & 1,07856 & 0,28078 \\
\hline $\mathrm{PETR}_{3}$ & Volatilidade $(\omega)$ & 0,411288 & 0,088410 & 4,652056 & $<0,001$ \\
\hline $\mathrm{BBAS}_{3}$ & Retorno $(\delta)$ & 0,11957 & 0,00815 & 14,67920 & $<0,001$ \\
\hline \multirow{2}{*}{ VALE5 } & Retorno $(\delta)$ & $-0,23161$ & 0,02192 & $-10,56630$ & $<0,0001$ \\
\hline & Volatilidade $(\omega)$ & 0,09413 & 0,01496 & 6,29240 & $<0,0001$ \\
\hline \multirow{2}{*}{ VALE3 } & Retorno $(\delta)$ & $-0,324164$ & 0,054404 & $-5,9585$ & $<0,0001$ \\
\hline & Volatilidade $(\omega)$ & 0,482791 & 0,225188 & 2,1439 & 0,032037 \\
\hline
\end{tabular}

Tabela 4. Resultados com intervalo de 10 dias pós-divulgação das notícias

\begin{tabular}{|c|c|c|c|c|c|}
\hline \multicolumn{2}{|l|}{ Ativo } & \multirow{2}{*}{$\begin{array}{c}\text { Estimativa } \\
-0,15743\end{array}$} & \multirow{2}{*}{$\begin{array}{c}\text { Erro padrão } \\
0,00878\end{array}$} & \multirow{2}{*}{$\begin{array}{c}\text { T-Value } \\
-17,92677\end{array}$} & \multirow{2}{*}{$\begin{array}{c}\text { P-Valor } \\
0,0001\end{array}$} \\
\hline PETR & Retorno $(\delta)$ & & & & \\
\hline 1) 4 & Volatilidade $(\omega)$ & 0,01664 & 0,00569 & 2,92612 & 0,00343 \\
\hline $\mathrm{PETR}_{3}$ & Volatilidade $(\omega)$ & 0,307617 & 0,090620 & 3,394593 & 0,000687 \\
\hline $\mathrm{BBAS}_{3}$ & Retorno $(\delta)$ & 0,13385 & 0,00764 & 17,51450 & $<0,0001$ \\
\hline \multirow{2}{*}{ VALE $5_{5}$} & Retorno $(\delta)$ & $-0,23259$ & 0,02293 & $-10,14450$ & $<0,0001$ \\
\hline & Volatilidade $(\omega)$ & 0,08908 & 0,01421 & 6,26870 & $<0,0001$ \\
\hline \multirow{2}{*}{ VALE3 } & Retorno $(\delta)$ & $-0,187544$ & 0,008857 & $-21,1758$ & $<0,0001$ \\
\hline & Volatilidade $(\omega)$ & 0,413990 & 0,197360 & 2,0976 & 0,035937 \\
\hline
\end{tabular}

Tabela 5. Resultados com intervalo de 15 dias pós-divulgação das notícias

\begin{tabular}{|c|c|c|c|c|c|}
\hline \multicolumn{2}{|l|}{ Ativo } & \multirow{2}{*}{$\begin{array}{l}\text { Estimativa } \\
-0,15163\end{array}$} & \multirow{2}{*}{$\begin{array}{c}\text { Erro padrão } \\
0,00835\end{array}$} & \multirow{2}{*}{$\begin{array}{c}\text { T-Value } \\
-18,16100\end{array}$} & \multirow{2}{*}{$\begin{array}{c}\text { P-Valor } \\
<, 0001\end{array}$} \\
\hline DETP, & Retorno $(\delta)$ & & & & \\
\hline PEIR4 & Volatilidade $(\omega)$ & 0,02003 & 0,00806 & 2,48590 & 0,01292 \\
\hline $\mathrm{PETR}_{3}$ & Volatilidade $(\omega)$ & 0,235711 & 0,090987 & 2,5906 & 0,00958 \\
\hline $\mathrm{BBAS}_{3}$ & Retorno $(\delta)$ & 0,13970 & 0,00734 & 19,04480 & $<0,0001$ \\
\hline \multirow{2}{*}{ VALE $_{5}$} & Retorno $(\delta)$ & $-0,23097$ & 0,02410 & $-9,58350$ & $<0,0001$ \\
\hline & Volatilidade $(\omega)$ & 0,08622 & 0,01390 & 6,20490 & $<0,0001$ \\
\hline \multirow{2}{*}{ VALE3 } & Retorno $(\delta)$ & $-0,144889$ & 0,073957 & $-1,9591$ & 0,050103 \\
\hline & Volatilidade $(\omega)$ & 0,019606 & 0,005226 & 3,7519 & 0,000175 \\
\hline
\end{tabular}

Iniciando com os resultados obtidos para a Petrobras, observa-se que, para todos os cenários, o comportamento das variáveis se manteve. Para as ações preferenciais (PETR4), a variável dependente, sendo entendida como prêmio pelo risco, apresentou queda para todos os intervalos de aparecimento da notícia, no qual o evento de corrupção observado afetou negativamente o prêmio pelo risco (e consequentemente o retorno ajustado) de maneira significante em um nível crítico considerado de $5 \%$. Quando analisada a medida de risco ligada ao ativo, ou seja, a volatilidade do prêmio pelo risco, 
encontramos o mesmo padrão para todos os cenários, no qual o evento provoca um aumento na percepção de risco da PETR4. O maior efeito foi observado para os intervalos de dois e cinco dias após a divulgação da notícia de corrupção, porém sendo somente o resultado da janela de dois dias estatisticamente relevante para análise em um nível crítico de $5 \%$. Quanto ao efeito para as ações ordinárias (PETR3), percebe-se que o evento não apresenta impacto estatístico significante para a variável dependente, porém é possível observar uma relação existente e significante para a volatilidade, na qual todos os cenários utilizados apresentaram um aumento expressivo para o risco do ativo, em média de 34\%, em um nível crítico de $5 \%$. Comparando o impacto na medida de risco para ações preferenciais (PETR4) e ordinárias (PETR3), é possível identificar um maior impacto do evento para o segundo caso, o que pode ser explicado pela baixa prioridade que os titulares desses ativos possuem para receber dividendos ou retornos sobre o investimento feito.

Ao contrário do efeito observado para o ativo ligado ao setor de petróleo, o ativo BBAS3 apresentou um aumento para ambas as variáveis em todos os cenários. Para todos os intervalos de dias pós-evento, teve-se um aumento no valor do prêmio pelo risco do Banco do Brasil, sendo estatisticamente relevante a $5 \%$. Uma possível explicação para a valorização das ações é que os agentes econômicos que atuam na bolsa brasileira estão movendo seu capital de ativos como a PETR4 para outros setores, mas ainda se mantendo sob o véu estatal. A volatilidade também apresentou comportamento positivo em relação ao evento, no qual, a cada notícia divulgada, o risco do BBAS3 apresentou um aumento, porém sendo relevante apenas para os cenários com intervalo maior que dois dias pós-divulgação da notícia de corrupção. Tal resultado reforça a hipótese da memória dos investidores em relação aos acontecimentos do mercado, sendo o maior valor da volatilidade observado na janela de cinco dias e sofrendo uma queda com o aumento da janela.

$\mathrm{O}$ ativo ligado à empresa Vale (VALE5) apresentou comportamento similar ao encontrado para PETR4. O retorno apresentou uma tendência negativa ao aparecimento do evento em todos os cenários e com relevância estatística, no qual se entende que o evento de corrupção resultou em uma desvalorização do preço de fechamento ajustado dia após dia. A medida de risco apresentou uma tendência positiva, na qual a percepção de risco da VALE5 aumenta a cada divulgação em todos os cenários, porém nota-se uma queda na volatilidade ao aumentarmos o intervalo pós-notícia, o que implica a "perda de memória" do investidor, assim como no caso do Banco do Brasil.

Além da cotação na bolsa brasileira, as empresas estudadas apresentam parte de seus recursos adquirida no mercado internacional. Uma vez que o presente estudo busca entender o impacto do evento no valor real e seu risco nas firmas, a identificação e análise da percepção do investidor externo ao Brasil é importante para mensurar como a corrupção institucional pública é interpretada e a reação da comunidade internacional diante do evento.

\section{Mercado internacional}

Da mesma maneira trabalhada para o cenário nacional, as tabelas a seguir apresentam o impacto do evento sobre ativos brasileiros negociados na bolsa norte-americana por meio das ADRs. Os ativos são referentes às mesmas firmas analisadas anteriormente:

\section{Tabela 6. Resultados internacionais sem intervalo pós-divulgação das notícias}

\begin{tabular}{|c|c|c|c|c|c|}
\hline \multicolumn{2}{|l|}{ Ativo } & $\begin{array}{l}\text { Estimativa } \\
-0,385367\end{array}$ & $\begin{array}{c}\text { Erro padrão } \\
0,022586\end{array}$ & $\begin{array}{l}\text { T-Value } \\
-17,062\end{array}$ & $\begin{array}{c}\text { P-Valor } \\
<0,0001\end{array}$ \\
\hline 政的 & Volatilidade $(\omega)$ & 0,000000 & 0,004873 & 0,000 & 1,000000 \\
\hline \multirow{2}{*}{ PBR } & Retorno $(\delta)$ & $-0,223702$ & 0,063765 & $-3,50821$ & 0,000451 \\
\hline & Volatilidade $(\omega)$ & 0,158795 & 0,206887 & 0,76755 & 0,442756 \\
\hline BDORY & Volatilidade $(\omega)$ & 0,001863 & 0,003463 & 0,53806 & 0,590537 \\
\hline \multirow{2}{*}{ VALE.P } & Retorno $(\delta)$ & $-0,405261$ & 0,024724 & $-16,3913$ & $<0,0001$ \\
\hline & Volatilidade $(\omega)$ & 0,037458 & 0,010913 & 3,4326 & 0,000598 \\
\hline
\end{tabular}


ARTIGOS | CORRUPÇÃO GOVERNAMENTAL NO MERCADO DE CAPITAIS: UM ESTUDO ACERCA DA OPERAÇÃO LAVA JATO

Ana Julia Akaishi Padula | Pedro Henrique Melo Albuquerque

Tabela 7. Resultados internacionais com intervalo de dois dias pós-divulgação das notícias

\begin{tabular}{|c|c|c|c|c|c|}
\hline \multicolumn{2}{|l|}{ Ativo } & \multirow{2}{*}{$\begin{array}{l}\text { Estimativa } \\
-0,302383\end{array}$} & \multirow{2}{*}{$\begin{array}{c}\text { Erro padrão } \\
0,066405\end{array}$} & \multirow{2}{*}{$\begin{array}{l}\text { T-Value } \\
-4,5536\end{array}$} & \multirow{2}{*}{$\begin{array}{l}\text { P-Valor } \\
<0,0001\end{array}$} \\
\hline PBRA & Retorno $(\delta)$ & & & & \\
\hline 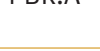 & Volatilidade $(\omega)$ & 0,081036 & 0,032606 & 2,4853 & 0,012945 \\
\hline \multirow{2}{*}{ PBR } & Retorno $(\delta)$ & $-0,231084$ & 0,039713 & $-5,8189$ & $<0,0001$ \\
\hline & Volatilidade $(\omega)$ & 0,060436 & 0,023479 & 2,5741 & 0,010050 \\
\hline BDORY & Retorno $(\delta)$ & $-0,336692$ & 0,016017 & $-21,0215$ & $<0,0001$ \\
\hline \multirow{2}{*}{ VALE.P } & Retorno $(\delta)$ & $-0,411128$ & 0,024559 & $-16,7403$ & $<0,0001$ \\
\hline & Volatilidade $(\omega)$ & 0,043638 & 0,008284 & 5,2679 & $<0,0001$ \\
\hline \multirow{2}{*}{ VALE } & Retorno (ס) & $-0,385960$ & 0,022855 & $-16,88741$ & $<0,0001$ \\
\hline & Volatilidade $(\omega)$ & 0,031614 & 0,010786 & 2,93115 & 0,003377 \\
\hline
\end{tabular}

Tabela 8. Resultados internacionais com intervalo de cinco dias pós-divulgação das notícias

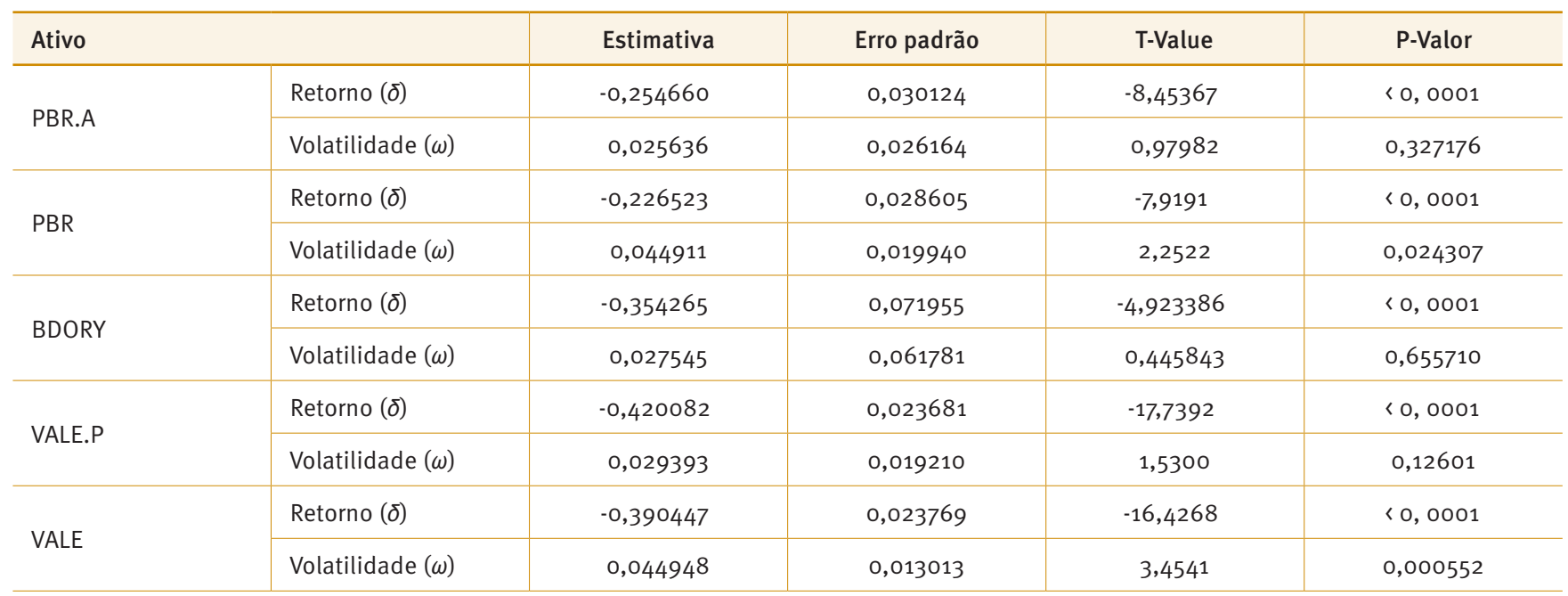

Tabela 9. Resultados internacionais com intervalo de 10 dias pós-divulgação das notícias

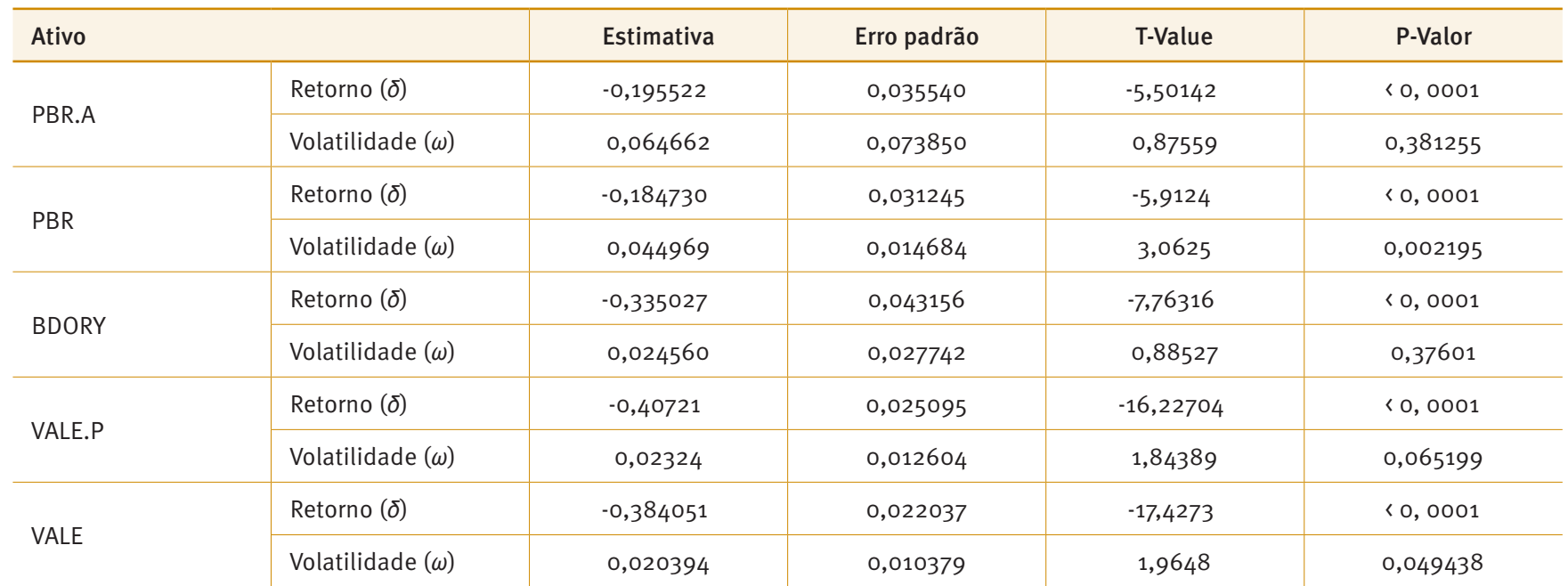


Tabela 10. Resultados internacionais com intervalo de 15 dias pós-divulgação das notícias

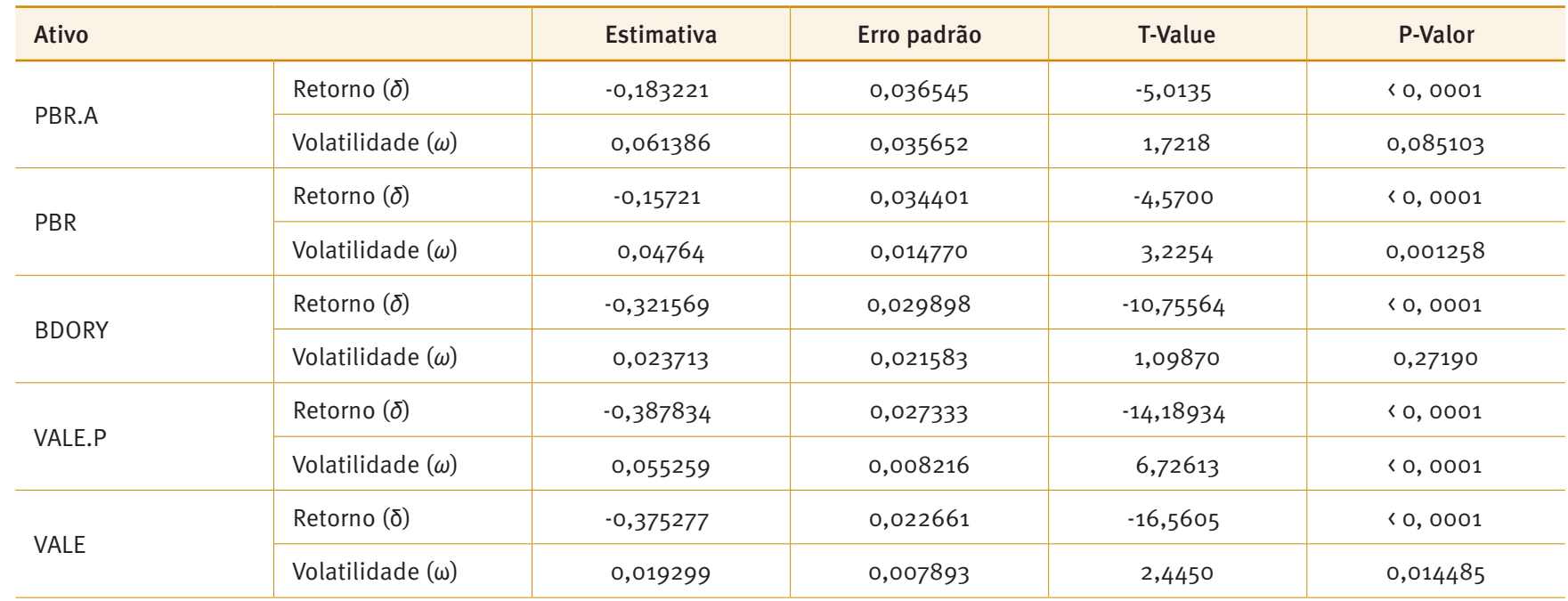

Os ativos da Petrobras negociados na bolsa norteamericana, preferenciais (PBR.A) e ordinários (PBR), apresentaram um comportamento semelhante para todos os cenários estimados. Independentemente do tipo de ação negociada, o prêmio pelo risco foi negativamente impactado pelo evento reportado, no qual se percebe uma forte reação do mercado para os primeiros dias seguida de uma amenização da desvalorização, em um nível crítico de $5 \%$ de confiança. Considerando todos os cenários estipulados, e considerando o efeito da memória do investidor, os ativos sofrem desvalorização média de $26,41 \%$ para PBR.A e $20,46 \%$ para PBR. Contudo, ao observar o efeito na volatilidade, somente as ações ordinárias apresentam um aumento no risco percebido pelo mercado. A PBR.A não apresentou em nenhum dos cenários alterações estatisticamente consideráveis, uma vez que ultrapassam o nível crítico de $5 \%$, enquanto a PBR apresenta resultados significativos posteriores à janela de dois dias da exposição da notícia aos agentes econômicos, o que pode representar o tempo de absorção e reação do mercado a um evento específico da realidade brasileira.

A ação BDORY do Banco do Brasil apresentou um comportamento similar ao da PBR, ambas sendo classificadas como ações ordinárias. 0 prêmio pelo risco sofreu desvalorização em todos os cenários, em média de 33,5\%. A volatilidade mostrou-se relevante apenas a partir da janela de dois dias de exposição da notícia, e, assim como observado para a PBR, é possível que exista um tempo de interpretação e reação do investidor estrangeiro para notícias brasileiras.

Os resultados da empresa Vale seguem a tendência apresentada pelos resultados da Petrobras. As ações preferenciais (VALE.P) e ordinárias (VALE) são afetadas de maneira negativa pelo evento, em média $40 \%$ para VALE.P e $38 \%$ para VALE, e dentro do nível crítico de análise $5 \%$, seguidas de uma suavização da desvalorização, o que indica que, com o passar do tempo, os investidores estrangeiros parecem absorver a notícia e amenizá-la. Em contrapartida, a percepção de risco apresentou um aumento de maneira geral para ambos os ativos em todos os cenários. É interessante notar o efeito para o caso da empresa Vale, no qual tanto o comportamento do prêmio pelo risco quanto o próprio risco apresentam uma curva similar de suavização com o passar do tempo; essa informação é um indício de que o mercado internacional apresenta um período de adaptação a um evento específico da política brasileira.

\section{CONCLUSÃO}

Obtidas as estimativas associadas ao impacto do evento para os ativos nas bolsas nacional e norte-americana, é interessante comparar a intensidade que o fenômeno apresentou para cada empresa.

A Petrobras possui, nos mercados brasileiro e norteamericano, ações classificadas como preferenciais (PETR4 e PBR.A) e ordinárias (PETR3 e PBR). Considerando a estimativa do impacto do evento na variável dependente, em todos os cenários construídos e utilizando apenas aqueles menores do que o nível crítico estabelecido, observou-se que o mercado internacional é mais impactado pelo evento do que o mercado nacional, no qual a PBR.A sofreu uma desvalorização de aproximadamente $-36,41 \%$ e a PBR -20,46\%, enquanto a PETR4 - $15,97 \%$ e a PETR3 não apresentou evidências de alteração estatisticamente significante em seu prêmio pelo risco. Quando comparados os riscos atribuídos aos ativos, percebe-se que, para ações ordinárias, 
existe um aumento significante no mercado internacional, porém, para ações ordinárias, o resultado foi o oposto. A PETR3 superou fortemente o reajuste do risco em relação à $\mathrm{PBR}$, resultado compreensível, uma vez que consideramos que não houve evidências para o impacto do evento sobre a PETR3.

O Banco do Brasil possui apenas ações ordinárias nos mercados em que é negociado, e apresentou comportamentos destoantes. Para o mercado brasileiro (BBAS3), foi encontrada uma valorização média de $12 \%$, considerando apenas os cenários cujo $p$-valor foi inferior a $5 \%$, enquanto para a bolsa norte-americana (BDORY) houve uma desvalorização média de $33,5 \%$ para as mesmas condições. A divergência entre o efeito do evento sobre o prêmio pelo risco pode ser um indício de que, para os investidores nacionais, o setor financeiro apresenta uma opção viável para direcionar seus recursos em meio aos escândalos políticos envolvendo a Petrobras, enquanto para o investidor estrangeiro não se percebe diferença entre os setores das empresas brasileiras, levando à desvalorização. A medida de risco foi positiva para BBAS3 e BDORY, porém com um efeito mais significativo para o cenário brasileiro do que no cenário internacional. 0 mais interessante nessa relação é que, mesmo com uma percepção de risco maior sobre o ativo no Brasil, os investidores continuam a se interessar e adquiri-lo, enquanto o mercado internacional aparenta evitar adquirir a ação.

Para os ativos ligados à Vale, os dois tipos de ações apresentaram as maiores desvalorizações no exterior. A bolsa americana apresentou desvalorizações médias de $40 \%$ para VALE.P e de $38 \%$ para VALE, enquanto o efeito no mercado nacional apresentou uma média de quase metade, sendo desvalorizada 22,63\% a VALE5 e 20,88\% a VALE3. Porém, para a análise de volatilidade, investidores nacionais atribuem um nível de risco sobre investimento muito maior do que o apresentado por investidores internacionais, efeito similar ao encontrado no ativo BBAS3, que apresentou um maior impacto na volatilidade do que no prêmio pelo risco. Tais evidências justificam a queda no nível de investimento do País, uma vez que, se considerarmos a exigência de um maior retorno sobre investimento para nações com suspeitas ou casos de corrupção pública comprovados, como apontado por Ciocchini et al. (2003) e Mauro (1995), o Brasil, ao não cumprir esse requisito e por não apresentar sinais de melhora ou mudança no cenário, tem por resultado a perda de investidores em ativos públicos.

Como é possível observar pelos resultados relatados, o Brasil apresentou, para a maioria dos casos, desvalorização de seus ativos e consequente perda de investimentos de origem nacional e internacional. Tal resposta do mercado diante da corrupção governamental é reflexo da dificuldade do País de oferecer uma rentabilidade para seus investidores, e contribui com a comprovação real da perda de capital e aumento do risco percebido pelos demais agentes econômicos, que resultará em um aumento na fuga de capitais caso a confiabilidade e credibilidade do Brasil não sejam restauradas.

A popularidade das discussões sobre o tema da corrupção e seus efeitos chama a atenção do mundo todo, devido aos impactos que esse fenômeno possui no funcionamento dos países. Como apresentado pelo World Economic Forum em 2016 espera-se que os países tomem atitudes proativas contra a corrupção, em especial para a América Latina, dados seus altos índices de percepção corrupção. Esta pesquisa apresentou resultados empíricos e significantes que comprovam os efeitos prejudiciais na economia em longo prazo e evidencia que, enquanto o cenário de escândalos não for solucionado, o Brasil perderá ainda mais recursos financeiros e terá dificuldades em se recuperar da crise econômica atual. O caráter inovador desta pesquisa recai sobre o alerta quantificado no setor financeiro, com previsões de perda em caso da permanência da situação atual, e, por apresentar um modelo pioneiro na análise de um grande evento de corrupção registrado no País, é exemplo e um incentivo para que novas pesquisas no campo sejam realizadas para auxiliar a desconstrução da rede de corrupção no Brasil.

Além disso, o presente trabalho contribui para a construção da literatura nacional sobre o tema de corrupção governamental aplicado à área de investimentos. O Brasil dispõe de diversas pesquisas que já iniciaram o debate do tema, como Silva (1994) e Brei (1996), que trouxeram conceitos, problematizações e soluções teóricas para a corrupção governamental seguidos por análises recentes ligadas à análise da correlação entre o evento da corrupção pública e seus efeitos no crescimento do PIB, apresentada por Marino, Soares, Luca e Vasconcelos (2016), e nos gastos públicos, analisada por Caldas, Costa e Pagliarussi (2016). Gehrke, Borba e Ferreira (2017) comprovam que, durante os últimos anos, incluso o período escolhido para análise deste trabalho, o Brasil vem ocupando espaço na mídia internacional e o tema ligado à corrupção pública também. Esse resultado revela que a reputação internacional e a atratividade do País decrescem ao longo do tempo, enquanto medidas e políticas de governabilidade não forem adotadas. Quando comparado com a recente literatura internacional, o principal foco de estudo identificado é o efeito da corrupção nos gastos públicos e seu efeito generalizado na economia formal e informal, como apresentado por Cooray e Schneider (2013) e Benfratello, Monte e Pennacchio (2015), mas sem uma abordagem profunda relacionada a investimentos, como apresentado por esta pesquisa. 
Por um olhar financeiro e empresarial, a pesquisa demonstra quantitativamente a importância do evento de corrupção no cálculo e projeção de investimentos no País. Devido a limitações de disponibilidade de dados e tempo, não foi possível identificar um indicador quantitativo para a corrupção, sendo justificado assim o uso da proxy de aparecimento das notícias, mas seria mais preciso para modelos de previsão e estimativa de retorno de investimentos englobar índices que mensurem e incorporem o risco. É válido o questionamento de que tal risco de corrupção já esteja incluso no risco sistêmico ou nas taxas de retorno exigidas, porém o presente estudo comprova que mesmo os modelos que teoricamente abordam a sensibilidade e percepção de risco do investidor, como o CAPM, não são capazes de captar por completo o efeito do evento no mercado de ações.

Finalmente, o objetivo da pesquisa foi verificar o impacto da corrupção no mercado financeiro e de capitais, o que pode ser aproximado do impacto no investimento interno e externo do Brasil. Como apresentado pela literatura, o estudo sobre a formação e as consequências do evento corrupção estatal são discutidas, mas sem aplicações no cenário brasileiro. Como uma forma de combater esse evento, por meio de políticas de compliance, e aumentar a eficiência e captação de recursos, seria interessante buscar as reais causas da corrupção para que seja possível traçar planos de combate a ela, com transparência política e eficiência do sistema público.

\section{REFERÊNCIAS}

Banco Central do Brasil. (2015). Focus - Relatório de mercado. Recuperado de http://www.bcb.gov.br/pec/GCl/PORT/readout/R20151030.pdf

Banco Central do Brasil. (2017). Histórico das taxas de juros 2000 a 2014. Recuperado de https://www.bcb.gov.br/Pec/Copom/Port/taxaSelic.asp

Bardhan, P. (1997). Corruption and development: A review of issues. Journal of Economic Literature, 35(3), 1320-1346.

Bayley, D. H. (1966). The effects of corruption in a developing nation. Western Political Quarterly, 19(4), 719-732. doi:10.2307/445147

Benfratello, L., Del Monte, A., \& Pennacchio, L. (2015). Corruption and public debt: an empirical analysis. University of Naples "Federico II" and CSEF, WP.

Binder, J. (1998). The event study methodology since 1969. Review of Quantitative Finance and Accounting, 11(2), 111-137. doi:10.1023/A:1008295500105

BM\&FBOVESPA. Índice Bovespa (Ibovespa). Composição da carteira 2000 a 2014. Recuperado de http://www.bmfbovespa.com.br/ pt_br/produtos/indices/indices-amplos/indice-ibovespa-ibovespa-composicao-da-carteira.htm
Brei, Z. A. (1996a). A corrupção: Causas, conseqüências e soluções para o problema. RAP-Revista de Administração Pública, 30(3), 103-115.

Brei, Z. A. (1996b). Corrupção: Dificuldades para definição e para um consenso. RAP-Revista de Administração Pública, 30(1), 64-77.

Caldas, O. V., Costa, C. M., \& Pagliarussi, M. S. (2016). Corrupção e composição dos gastos governamentais: Evidências a partir do Programa de Fiscalização por Sorteios Públicos da Controladoria-Geral da União. RAP-Revista de Administração Pública, 50(2), 237-264. doi:10.1590/0034-7612140185

Campbell, J. Y., Lo, A. W. C., \& MacKinlay, A. C. (1997). The econometrics of financial markets. Princeton, NJ: Princeton University Press.

Ciocchini, F., Durbin, E., \& Ng, D. T. (2003). Does corruption increase emerging market bond spreads? Journal of Economics and Business, 55(5-6), 503-528. doi:10.1016/S0148-6195(03)00052-3

Claessens, S., Klingebiel, D., \& Schmukler, S. L. (2006). Stock market development and internationalization: Do economic fundamentals spur both similarly? Journal of Empirical Finance, 13(3), 316-350. doi:10.1016/j.jempfin.2006.03.002

Cooray, A., \& Schneider, F. (2013). How does corruption affect public debt? An empirical analysis (Working Paper No. 1322). Department of Economics, Johannes Kepler University of Linz.

Cox, D. R., \& Reid, N. (2004). A note on pseudolikelihood constructed from marginal densities. Biometrika, 91(3), 729-737.

Dann, L. Y. (1981). Common stock repurchases: An analysis of returns to bondholders and stockholders. Journal of Financial Economics, 9(2), 113-138. doi:10.1016/0304-405X(81)90010-6

Dobson, S., \& Ramlogan-Dobson, C. (2012). Why is corruption less harmful to income inequality in Latin America? World Development, 40(8), 1534-1545. doi:10.1016/j.worlddev.2012.04.015

Gehrke, G., Borba, J. A., \& Ferreira, D. M. (2017). A repercussão da corrupção brasileira na mídia: Uma análise comparada das revistas Der Spiegel, L 'Obs, The Economist, Time e Veja. RAP-Revista de Administração Pública, 51(1), 157-167. doi:10.1590/0034-7612158681

Hansen, P. R., \& Lunde, A. (2005). A forecast comparison of volatility models: Does anything beat a GARCH $(1,1)$ ? Journal of Applied Econometrics, 20(7), 873-889. doi:10.1002/jae.800

Huntington, S. P. (2006). Political order in changing societies. New Haven, CT: Yale University Press.

Jong, F. De, Kemna, A., \& Kloek, T. (1992). A contribution to event study methodology with an application to the Dutch stock market. Journal of Banking \& Finance, 16(1), 11-36. doi:10.1016/0378-4266(92)90076-C

Kurer, O. (1993). Clientelism, corruption, and the allocation of resources. Public Choice, 77(2), 259-273.

Lambsdorff, J. G. (2003). How corruption affects productivity. Kyklos, 56(4), 457-474. doi:10.1046/j.0023-5962.2003.00233.x

Lau, C. K. M., Demir, E., \& Bilgin, M. H. (2013). Experience-based corporate corruption and stock market volatility: Evidence from emerg. ing markets. Emerging Markets Review, 17, 1-13. doi:10.1016/j.ememar.2013.07.002

Leff, N. H. (1964). Economic development through bureaucratic corruption. American behavioral scientist, 8(3), 8-14.

Leys, C. (1965). What is the problem about corruption? The Journal of Modern African Studies, 3(2), 215-230. 
Ljung, G. M., \& Box, G. E. (1978). On a measure of lack of fit in time series models. Biometrika, 65(2), 297-303. doi:10.2307/2335207

Marino, P. D. B. L. P., Soares, R. A., Luca, M. M. M. De, \& Vasconcelos, A. C. de. (2016). Indicadores de governança mundial e sua relação com os indicadores socioeconômicos dos países do Brics. RAP-Revista de Administração Pública, 5o(5), 721-744.

Mauro, P. (1995). Corruption and growth. The Quarterly Journal of Economics, 110 (3), 681-712. doi:10.2307/2946696

Myrdal, G. (1994). Asian drama: An inquiry into the poverty of nations. New York: Twentieth Century Fund.

Nasdaq. (s.d.). Historical quote. Banco do Brasil S.A. ADR (Sponsored) Historical Stock Prices 2000 to 2014. Recuperado de http://www. nasdaq.com/symbol/bdory/historical.

Nasdaq. (s.d.). Historical quote. Petroleo Brasileiro S.A. Petrobras American Depositary Shares Historical Stock Prices 2000 to 2014 . Recuperado de http://www.nasdaq.com/symbol/pbr.a/historical.

Nasdaq. (s.d.). Historical quote. Petroleo Brasileiro S.A. Petrobras Common Stock Historical Stock Prices 2000 to 2014. Recuperado de http://www.nasdaq.com/symbol/pbr/historical.

Nasdaq. (s.d.). Historical quote. VALE S.A. American Depositary Shares Each Representing one common share Historical Stock Prices 2000 to 2014. Recuperado de http://www.nasdaq.com/symbol/vale/his torical.

Nasdaq. (s.d.). Historical quote. VALE S.A. Historical Stock Prices 2000 to 2014. Recuperado de http://www.nasdaq.com/symbol/vale.p/ historical.

Nasdaq. (s.d.). Indices. Major Stock Indices 2000 to 2014. Recuperado de http://www.nasdaq.com/markets/indices/major-indices.aspx.

Nguyen, T. T., \& Dijk, M. A. Van. (2012). Corruption, growth, and governance: Private vs. state-owned firms in Vietnam. Journal of Banking \& Finance, 36(11), 2935-2948. doi:10.1016/j.jbankfin.2012.03.027
Policia Federal. (2014). Operação Lava Jato - Fases. Recuperado de http://www.pf.gov.br/agencia/noticias/operacao-Lava Jato-fases

Rachev, S., \& Mittnik, S. (2000). Stable Paretian models in finance. New York, NY: Wiley.

Rose-Ackerman, S. (1997). The political economy of corruption. Corruption and the Global Economy, 31, 60.

Shleifer, A., \& Vishny, R. W. (1993). Corruption. The Quarterly Journal of Economics, 108(3), 599-617. doi:10.2307/2118402

Silva, M. (1994). Corrupção: Tentativa de uma definição funcional. RAP-Revista de Administração Pública, 28(1), 18-23.

Staikouras, S. K. (2006). Testing the stabilization hypothesis in the UK short-term interest rates: Evidence from a GARCH-X model. The Quarterly Review of Economics and Finance, 46(2), 169-189. doi:10.1016/j. qref.2005.04.003

United States Department of the Treasury. Resource Center. Daily Treasury Bill Rates Data 2000 to 2014. Recuperado de https://www.treasury.gov/resource-center/data-chart-center/interest-rates/Pages/ TextView.aspx?data=billratesAll

White, H. (1982). Maximum likelihood estimation of misspecified models. Econometrica, 50(1), 1-25. doi:10.2307/1912526

World Bank. (2015). World Development Indicators. Brazil. Recuperado de http://databank.worldbank.org/data/reports.aspx?. source $=2 \&$ country $=$ BRA

World Economic Forum. (2016a). Can Latin America put an end to corruption? Recuperado de https://www.weforum.org/agenda/2016/03/ can-latin-america-put-an-end-to-corruption

World Economic Forum. (2016b). Corruption in Latin America is skyrocketing: Here's why that's good news. Recuperado de https://www.weforum.org/agenda/2016/06/corruption-in-latin-america 